\title{
Evidence of wolframite-type structure in ultrasmall nanocrystals with a targeted composition $\mathrm{MnWO}_{4}$
}

Pascaline Patureau ${ }^{1}$, Rémi Dessapt ${ }^{1}$, Pierre-Emmanuel Petit ${ }^{1}$, Gautier Landrot ${ }^{2}$, Christophe Payen ${ }^{1, *}$, Philippe Deniard ${ }^{1, *}$

${ }^{1}$ Institut des Matériaux Jean Rouxel (IMN), Université de Nantes, CNRS, 2 rue de la Houssinière, BP 32229, 44322 Nantes cedex 3, France

${ }^{2}$ Synchrotron SOLEIL, F-91192 Gif sur Yvette, France

*Authors to whom inquiries regarding the paper may be addressed 
ABSTRACT: Here, we report a study of white-ochre powders with targeted composition $\mathrm{MnWO}_{4}$ prepared via a coprecipitation method. Through X-ray total scattering combined with pair distribution function analysis and Rietveld refinement of X-ray diffraction data we find that their crystal structure is similar to that of bulk-MnWO , despite a mean crystallite size of 1.0-1.6 nm and a significant deviation of the average chemical composition from $\mathrm{MnWO}_{4}$. The chemical formula derived from elemental and thermogravimetric analyses is


that $\mathrm{Mn}$ and $\mathrm{W}$ have the same oxidation states as in $\mathrm{MnWO}_{4}$. No magnetic ordering or spin glass or superparamagnetic behavior is observed above $2 \mathrm{~K}$, unlike in the case of $\mathrm{MnWO}_{4}$ nanocrystals having mean size higher than $10 \mathrm{~nm}$. 


\section{— INTRODUCTION}

Transition metal tungstates of the $\mathrm{MWO}_{4}$ type $(\mathrm{M}=\mathrm{Mn}, \mathrm{Fe}, \mathrm{Co}, \mathrm{Ni}, \mathrm{Cu}$, or $\mathrm{Zn})$ have attracted great interest due to potential applications in various fields, including humidity sensors ${ }^{1}$, multiferroic materials ${ }^{2}$, photocatalysts ${ }^{3}$, heterogeneous catalysts $^{4}$, biomedical imaging 5 , energy storage materials ${ }^{6}$, and color pigments $^{7}$. These compounds contain M(II) and tungsten(VI) cations in octahedral environments. They crystallize with the wolframite (monoclinic) structure ${ }^{8}$, with the exception of $\mathrm{CuWO}_{4}$ which exhibits a lower (triclinic) crystal symmetry associated with cooperative Jahn-Teller distortions of the $\mathrm{Cu}^{2+}\left(d^{9}\right)$ environment ${ }^{9}$. Crystal structures comprise infinite zigzag $\left(\mathrm{MnO}_{4}\right)_{\infty}$ and $\left(\mathrm{WO}_{4}\right)_{\infty}$ chains, running parallel to the same direction, of either edge-sharing $\mathrm{MO}_{6}$ distorted octahedra or edge-sharing $\mathrm{WO}_{6}$ distorted octahedra. ${ }^{8-10}$ Their interesting properties have been investigated at different length scales, from bulk in single crystals or fine particles to nanoscale in nanosized materials. For applications or studies at the nanoscale, a possible key challenge is keeping crystal structures with high crystallinity and their associated bulk properties when decreasing particle size. For $\mathrm{MnWO}_{4}$, which is a well-studied multipurpose material ${ }^{1,2,5,6}$, phase-pure nanoparticles of controlled size and shape have been prepared by mild solution methods $^{11,12}$, and single-crystal nanoparticles with mean size in the range of 10-50 nm have been studied ${ }^{13-15}$. When looking at their structure-dependent bulk electronic properties, one can note that magnetic transitions associated with type-II multiferroicity in bulk-MnWO 4 are still visible in temperature-dependent magnetic susceptibility curves of $\approx 50 \mathrm{~nm}$ single-crystal nanopellets and nanorods ${ }^{15}$ but are absent when mean crystallite size is $\approx 10 \mathrm{~nm}^{14}$.

Here, we address the problem of how the wolframite structural order of $\mathrm{MnWO}_{4}$ evolves when downscaling towards the ultrasmall nanocrystallite range $(\leq 5-10 \mathrm{~nm})$ where unique physicochemical properties are generally observed ${ }^{16}$. This unresolved issue also deserved attention with regards to the chemistry of transition metal tungstate nanoparticle 
formation. ${ }^{17}$ In this work, we have studied the crystal structure and metal oxidation states in ultrasmall nanocrystals with targeted composition $\mathrm{MnWO}_{4}$ through $\mathrm{X}$-ray total scattering combined with pair distribution function analysis (PDF), X-ray diffraction (XRD), X-ray Absorption Near Edge Structure (XANES) spectroscopy, and magnetic susceptibility measurements. Samples were prepared by using a coprecipitation method that produces a white-ochre solid. Although the formation of a white-ochre precipitate with unknown chemical formula has already been observed in a few previous works ${ }^{18,19}$ which employed a co-precipitation method, no characterization data has been reported so far. We therefore have also determined the actual macroscopic chemical formula and have examined the thermal stability of these precipitates in air by variable temperature XRD.

\section{— EXPERIMENTAL SECTION}

Synthesis, Elemental and Thermogravimetric Analyses. High-purity reagent chemicals were purchased from commercial vendors. Elemental analyses (Mn, W, Na, and H contents) were performed on as-prepared powders at the "Service Central d'Analyse du CNRS", Vernaison, France. Thermogravimetric measurements were performed in flowing air. Powders were placed in alumina crucibles and heated up to $800^{\circ} \mathrm{C}$ at $3 \mathrm{~K} / \mathrm{min}$.

Characterization. Attempts at carrying out transmission electron microscopy experiments failed because samples were unstable. X-ray diffraction (XRD) patterns were collected at room temperature on a Bruker D8 Advance instrument using monochromatic $\mathrm{Cu}_{\mathrm{K}-\mathrm{L} 3}(\lambda=$ $1.540598 \AA ̊$ ) X-rays and a LynxEye detector. Temperature variable XRD experiments were carried out on a Bruker D8 Advance instrument using CuKL2-L3 $(\lambda=1.540598,1.54433 \AA)$ radiation and a Våntec detector. Le Bail and Rietveld analyses of the XRD data were 
performed using JANA $2006^{20}$ and the Cheary-Coelho fundamental approach for XRD profile parameters $^{21}$.

X-ray Absorption Spectroscopy analyses were done at SAMBA beamline, Synchrotron SOLEIL. The storage ring was operated with multi-bunch and top-up modes, with $2.75 \mathrm{GeV}$ electron energy and $450 \mathrm{~mA}$ current. Powder samples were pelletized prior acquisition and analyzed at room temperature. X-ray Absorption Near Edge Structure (XANES) spectra were collected at the $\mathrm{W} \mathrm{L}_{3}$ edge (10207 eV) and Mn K edge (6539 eV) in fluorescence mode using a Si(220) monochromator, a Ge multi-pixel fluorescence detector (Canberra), and step-bystep acquisition mode. At each edge, multiple spectra were collected until the signal-to-noise ratio of the corresponding merge spectrum was satisfactory. All spectra were averaged, normalized and corrected from self-absorption effects using Athena featured in Demeter Software Package. ${ }^{22}$

X-ray total scattering measurements were performed at room temperature on a Bruker D8 Advance instrument using Мокц2-L3 $(\lambda=0.70929,0.71358 \AA$ ) radiation and a Våntec detector. This set up produces, for a $2 \theta$ range up to $150^{\circ}$, a $Q_{\max }$ of $17 \AA^{-1}$. Observed PDFs were calculated using PDFgetX2 software ${ }^{23}$ and real space refinements were performed using PDFgui $^{24}$.

A Quantum Design MPMS-XL7 magnetometer was used to collect temperature-dependent DC magnetization data. Zero field cooled (ZFC) and field cooled (FC) magnetization measurements were taken from 2 to $300 \mathrm{~K}$ in an applied field of $\mu_{0} H=0.01$ or $0.1 \mathrm{~T}$. Data were corrected for the diamagnetism of the sample holder as well as for core diamagnetism using Pascal's constants ${ }^{25}$. 


\section{— RESULTS AND DISCUSSION}

Synthesis and Compositional Analysis. Samples with a targeted Mn:W molar ratio of 1.0 were prepared in a reproducible manner by using a coprecipitation method. ${ }^{26}$ Aqueous solution of manganese chloride $\mathrm{MnCl}_{2} .4 \mathrm{H}_{2} \mathrm{O}(0.6 \mathrm{mmol}$ in $10 \mathrm{~mL})$ was added slowly to an aqueous solution of $\mathrm{Na}_{2} \mathrm{WO}_{4} .2 \mathrm{H}_{2} \mathrm{O}(0.6 \mathrm{mmol}$ in $10 \mathrm{~mL})$ at room temperature under vigorous magnetic stirring, leading to immediate precipitation of a white-ochre solid. The $\mathrm{pH}$ was kept constant at about 7 by adding manually $\mathrm{HCl} 0.1 \mathrm{M}$ and the slurry was stirred at room temperature for five minutes. After filtration, the powder was washed with water, ethanol, and dried in air at room temperature. The final yield in W was 22 mol\%. Elemental analyses showed no evidence for incorporation of sodium cations and were in good agreement with the chemical composition $\mathrm{H}_{6.4} \mathrm{Mn}_{0.8} \mathrm{WO}_{7.0}$ that fulfills electroneutrality (see the Supporting Information). Thermogravimetric data are shown in Figure 1. A total mass loss of about $19 \%$ took place in three steps in the temperature range of $25-800{ }^{\circ} \mathrm{C}$. The small mass loss of $\approx 1.5$ $\%$ observed below $\approx 50{ }^{\circ} \mathrm{C}$ is likely due to the removal of free and/or physisorbed water. Thermogravimetric data also revealed a mass loss of $\approx 15.5 \%$ in the temperature range of 50 $150^{\circ} \mathrm{C}$ and $\approx 2 \%$ in the temperature range of $150-800{ }^{\circ} \mathrm{C}$. By rewriting the chemical formula


losses of $15.5 \%$ and $2 \%$ correspond to the losses of $3 \mathrm{H}_{2} \mathrm{O}$ and $0.4(\mathrm{OH})$, respectively.

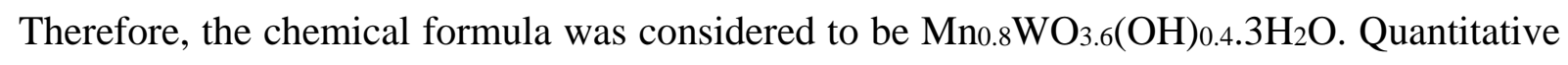
analysis of the $\mathrm{XRD}$ pattern at $700^{\circ} \mathrm{C}$ and magnetic susceptibility data were also fully consistent with this formula as described hereafter. The low Mn:W ratio in the precipitate (0.8) agrees with the results in ref. 26 and implies the presence of unreacted Mn(II) cations in


nanopellets when the white-ochre slurry is hydrothermally treated at $180^{\circ} \mathrm{C}^{15}$. 


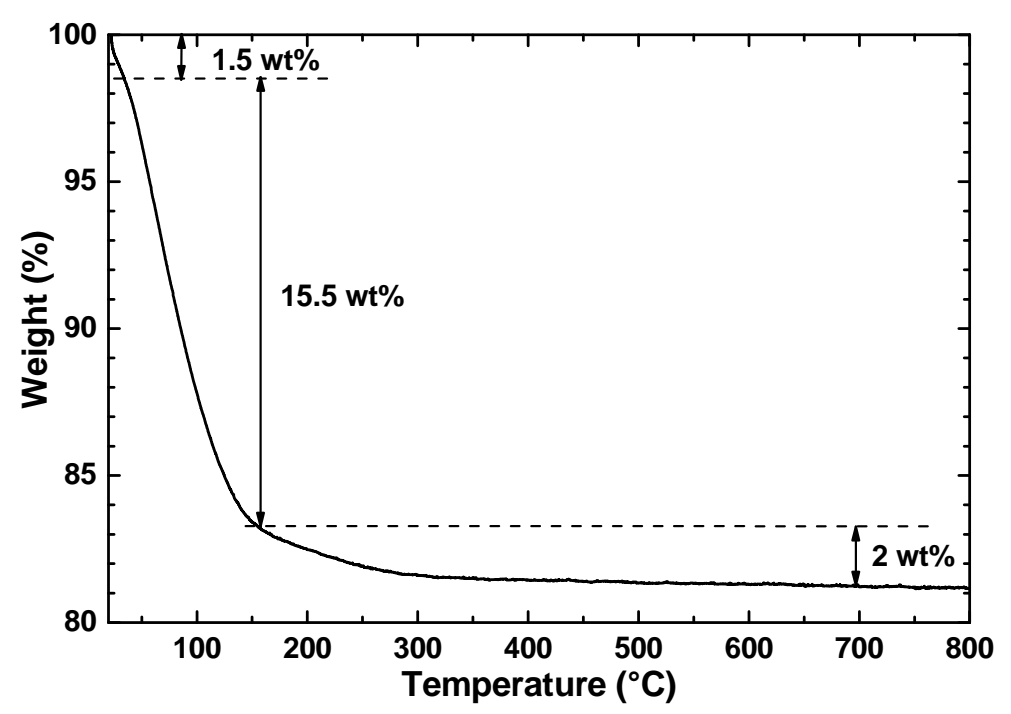

Figure 1. Thermogravimetric curve for $\mathrm{Mn}_{0.8} \mathrm{WO}_{3.6}(\mathrm{OH})_{0.4 .} 3 \mathrm{H}_{2} \mathrm{O}$ in flowing air.

Variable Temperature X-ray Diffraction. Figure $2\left(25^{\circ} \mathrm{C}\right)$ presents the room temperature XRD pattern of as-prepared powder. No distinct Bragg reflections was observed and thus the compound could at first sight be considered to be amorphous. Three very broad features centered at $2 \theta \approx 30,55$, and $65^{\circ}$ were however visible in the XRD pattern and the absence of narrow Bragg reflections could instead be due to strong peak broadening and overlap. Syntheses of powder samples of $\mathrm{MnWO}_{4}$ via co-precipitation are usually followed by a brief heating in air at a moderate temperature, e.g., $400-500{ }^{\circ} \mathrm{C}$, that makes it possible to observe narrow Bragg reflections in XRD patterns ${ }^{26}$. Figure 2 also shows the evolution of the diffraction pattern as a function of temperature. At $450{ }^{\circ} \mathrm{C}$, the diffraction pattern was similar to that of $\mathrm{MnWO}_{4}$ (ICSD \# 67907). Above $450{ }^{\circ} \mathrm{C}$ small additional peaks appeared. They were observed up to $700{ }^{\circ} \mathrm{C}$ as evidenced by the inset of Figure 2 where they are marked with asterisks. They correspond to the krasnogorite variety of $\mathrm{WO}_{3}$ (pdf file 71-0131). A twophase refinement carried out by the Rietveld method for $700^{\circ} \mathrm{C}$ (see Figure S1 in the Supporting Information), leaded to $14.8+/-1.2 \mathrm{wt} \%$ of $\mathrm{WO}_{3}$ in perfect agreement with









Patterns were collected in air at several temperatures from 25 to $700{ }^{\circ} \mathrm{C}$ (temperature is indicated below the corresponding pattern) using the same powder for the whole data set. The inset shows a portion of the pattern recorded at $700^{\circ} \mathrm{C}$. Bragg reflections marked with asterisks are from $\mathrm{WO}_{3} . h k l$ indices are those of $\mathrm{MnWO}_{4}$ (ICSD 67907).

X-ray Absorption Near Edge Structure (XANES). XANES was used in order to determine




well characterized micrometric powder of $\mathrm{MnWO}_{4}$. This figure also shows our W $\mathrm{L}_{3} \mathrm{XANES}$ data for the reactant $\mathrm{Na}_{2} \mathrm{WO}_{4} \cdot 2 \mathrm{H}_{2} \mathrm{O}$, which contains $\mathrm{W}(\mathrm{VI})$ ions in tetrahedral environment. ${ }^{27}$ For each edge, spectra were collected under the same experimental conditions. The ionic state and local environment of $\mathrm{W}$ can be identified by comparing the onset energies and shapes of the W $L_{3}$ spectra. ${ }^{28}$ From data in Figure 3, it is evident that tungsten is exclusively found in the form of W(VI) in octahedral environment, just like in $\mathrm{MnWO}_{4}$. For the $\mathrm{Mn} K$ edge data, 



Figure 3. XANES spectra for $\mathrm{Mn}_{0.8} \mathrm{WO}_{3.6}(\mathrm{OH})_{0.4 .} 3 \mathrm{H}_{2} \mathrm{O}$ (termed Precipitate) and for micrometric powder of $\mathrm{MnWO}_{4}$. Top panel: $\mathrm{W} \mathrm{L}_{3}$-edge spectra. Data for $\mathrm{Na}_{2} \mathrm{WO}_{4} \cdot 2 \mathrm{H}_{2} \mathrm{O}$ were also recorded for the sake of comparison. Bottom panel: Mn $K$ edge spectra. The arrow indicates pre-edge features at $\approx$ $6540 \mathrm{eV}$ which are related to electronic transitions to $1 s$ core levels to empty hybridized $3 d / 4 p$ states.

determination of the Mn oxidation state from the main edge energy is not possible due to single and multiple scattering effects. ${ }^{29}$ However, the weak pre-edge features observed at


of $\mathrm{Mn}(\mathrm{II})$ in octahedral symmetry. ${ }^{29}$

Pair Distribution Function (PDF) Analysis. A PDF analysis of X-ray total scattering data was then performed to study the interatomic distances $r$. Total scattering structure factors


the Supporting Information to visualize the quality of the data treatment. Figure 4 shows the




to those observed in the PDF of a micrometric powder sample of $\mathrm{MnWO}_{4}$, which is also shown in Figure 4. The calculated PDF of the published wolframite structure of bulk $\mathrm{MnWO}_{4}$ (ICSD \# 67907) $)^{10}$ is presented in Figure 5 for the sake of comparison. For


metal-oxygen distances in $\mathrm{WO}_{6}$ and $\mathrm{MnO}_{6}$ octahedra of the bulk- $\mathrm{MnWO}_{4}$ structure, respectively. As in bulk-MnWO 4 , a weak peak at $\approx 2.9 \AA$ can be associated with oxygenoxygen distances. The large and intense peak at $3.6 \AA$ is likely due to the shortest metal-metal distances; Mn-Mn or $\mathrm{W}-\mathrm{W}$ distances for edge-sharing $\mathrm{MnO}_{6}$ or $\mathrm{WO}_{6}$ octahedra that form the $\left(\mathrm{MnO}_{4}\right)_{\mathrm{n}}$ or $\left(\mathrm{WO}_{4}\right)_{\mathrm{n}}$ chains, and $\mathrm{Mn}-\mathrm{W}$ distances for two $\mathrm{MnO}_{6}$ and $\mathrm{WO}_{6}$ octahedra sharing a corner. Furthermore, the two peaks found between 4 and $5 \AA$ match with the $\mathrm{W}$-W distances of 4.45 and $4.8 \AA$ in bulk-MnWO 4 . All these observations prompted us to perform a


model with a size damping envelope. The obtained fit, shown in Figure 4, was of good quality up to about $6 \AA$.








Figure 4. Experimental PDF $G(r)$ for $\mathrm{Mn}_{0.8} \mathrm{WO}_{3.6}(\mathrm{OH})_{0.4} \cdot 3 \mathrm{H}_{2} \mathrm{O}$ (top panel) and for a micrometric powder sample of $\mathrm{MnWO}_{4}$ (bottom panel) derived from X-ray total scattering data taken with Mo $\mathrm{K} \alpha 1 \mathrm{~K} \alpha 2$ radiations (blue dots). The red lines show fits obtained using (i) a $\mathrm{MnWO}_{4}$ particle model in the case of $\mathrm{Mn}_{0.8} \mathrm{WO}_{3.6}(\mathrm{OH})_{0.4} \cdot 3 \mathrm{H}_{2} \mathrm{O}$ (ii) the published wolframite structure model of bulk- $\mathrm{MnWO}_{4}$ in the case of micrometric $\mathrm{MnWO}_{4}$. The lower green curves show the differences between the PDF and the refinement.

Figure 5. Calculated PDF $G(r)$ of the published wolframite bulk-MnWO 4 model in the low $r$-region. Some interatomic distances are shown above or below the PDF. The five shortest W-W distances are marked with numbers ( 1 to 5 ) and are illustrated on the perspective views of the crystal structure shown below the graph.

Difficulty in refining the PDF for longer distances correlates with the ultrasmall fitted particle size, $1.3 \pm 0.3 \mathrm{~nm}$. A spherical nanocrystal of $\mathrm{MnWO}_{4}$ with a diameter of $1.3 \mathrm{~nm}$ would consist of about 8 unit cells and $\approx 100$ atoms, a significant number of which would be located on the surface. Deviation from the bulk $\mathrm{MnWO}_{4}$ composition, which includes a lower Mn-toW ratio of 0.8 and the incorporation of hydroxides into particle structure, could also explain differences in local structure.

Rietveld refinement and simulation of room-temperature XRD data. According to our


consistent with ultrasmall $\approx 1 \mathrm{~nm}$ nanocrystallites having a $\mathrm{MnWO}_{4}$-type structure. Thus, we performed a Rietveld refinement using the published bulk-MnWO 4 structural model. ${ }^{10}$ Given the low signal-to-noise ratio and the large width of Bragg reflections, only the following parameters were refined: scale factor, crystallite size and background (5-term Legendre polynomial). All $\mathrm{MnWO}_{4}$ structural parameters (taken from ICSD \# 67907) were held fixed. The refinement converged to obtain the results shown in Figure 6, which were in very good agreement with a nanoscale $\mathrm{MnWO}_{4}$-like compound. The refined crystallite size $0.82(2) \mathrm{nm}$ compares well with the value 1.3(3) nm derived from PDF analysis, given that microstrains could not be taken into account in the contribution to line broadening. An additional clue


(Supporting Information). In this latter figure, the X-ray diffraction pattern of $\mathrm{MnWO}_{4}$ (ICSD \# 67907) was simulated for two different crystallite sizes (50 and $1 \mathrm{~nm}$ ) and for CuK-L3 radiation. In the $1 \mathrm{~nm}$ case, the simulated pattern, which is very similar to that shown in 
Figure 6, results solely from overlap of many Bragg reflections and does not correspond to that of an amorphous material.

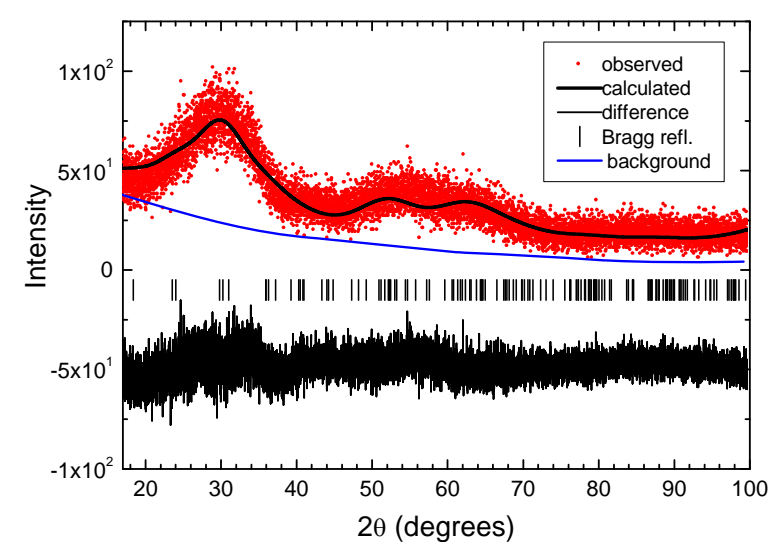

Figure 6. Final Rietveld refinement plot of the room-temperature XRD data (taken with $\mathrm{Cu}_{\mathrm{K}-\mathrm{L} 3}$ radiation) for $\mathrm{Mn}_{0.8} \mathrm{WO}_{3.6}(\mathrm{OH})_{0.4 .} 3 \mathrm{H}_{2} \mathrm{O}$. This refinement was performed using the published bulk$\mathrm{MnWO}_{4}$ structural model. The blue line shows the fitted background. Each hkl reflection, as represented by a vertical bar, overlaps with its neighbors, leading to a few broad features in the pattern.

Magnetic susceptibility. Finally, we turn to the magnetic properties. Temperature dependent magnetic susceptibility data, $\chi(T)$, of as-prepared powders were collected from 2 to $300 \mathrm{~K}$. As can be seen in Figure 7, the magnetic susceptibility obeys a Curie-Weiss law above $\approx 25 \mathrm{~K}$.


actually the one expected for 0.8 mole of $\mathrm{Mn}(\mathrm{II})$ ions having $S=5 / 2$ spin and for nonmagnetic W(VI) ions. Data in Figure 8 also indicate the absence of magnetic orders or superparamagnetic or spin-glass behaviors as the susceptibility keeps rising down to the base temperature (with no difference between the data measured under zero-field-cooled and that measured under field-cooled conditions). In bulk-MnWO 4 , three magnetic structures have been observed below $T_{\mathrm{N}}=13.5 \mathrm{~K}, T_{2}=12.5 \mathrm{~K}$ and $T_{1} \approx 8 \mathrm{~K} .{ }^{30}$ The two transitions at $T_{\mathrm{N}}$ and 
$T_{2}$ and the associated ferroelectric transition were still observed in nanocrystals with mean diameter of $50 \mathrm{~nm}^{15}$, but only one magnetic transition was visible at $6 \mathrm{~K}$ in nanocrystals with mean size of $10 \mathrm{~nm} .{ }^{14}$ In the multiferroic state of bulk-MnWO 4 below $T_{2}$, the incommensurate magnetic structure has a large magnetic unit cell volume of $2.8 \mathrm{~nm}^{3}$, which reflects the existence of long-ranged magnetic interactions. ${ }^{30,31}$ Therefore a loss of spin-driven multiferroicity is expected in any $\mathrm{MnWO}_{4}$ nanocrystallite having a volume lower than or comparable with the bulk magnetic cell volume. Deviation from $\mathrm{MnWO}_{4}$ composition should





Figure 7. Inverse molar magnetic susceptibility $1 / \chi$ versus temperature $T$ for $\mathrm{Mn}_{0.8} \mathrm{WO}_{3.6}(\mathrm{OH})_{0.4 \cdot} 3 \mathrm{H}_{2} \mathrm{O}$. The solid line corresponds to a Curie-Weiss behavior, $1 / \chi=(T-\theta) / C$, with $C$





Figure 8. Magnetic susceptibility $\chi(T)$ in the low- $\mathrm{T}$ region, $\mathrm{T} \leq 20 \mathrm{~K}$, for $\mathrm{Mn}_{0.8} \mathrm{WO}_{3.6}(\mathrm{OH})_{0.4 .3} \mathrm{H}_{2} \mathrm{O}$ 


\section{- CONCLUDING REMARKS}

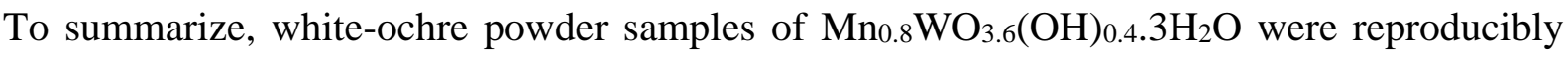
obtained by a simple salt metathesis reaction involving stoichiometric amounts of manganese(II) chloride and sodium tungstate dihydrate. A common belief is that this coprecipitation method gives dispersions containing amorphous particles which would act as precursors for the hydrothermal synthesis of crystallized nanoparticles of transition metal tungstates. ${ }^{13}$ However our study indicates that coprecipitation can lead to the formation of ultrasmall nanocrystals which retain important structural features of the bulk when synthesis conditions are chosen so as to prevent the formation of polyoxometalates in solution. In this case, there are similarities in the crystal structures between the precursor and the final nanosized $\mathrm{MnWO}_{4}$ material.

\section{- AUTHOR INFORMATION}

\section{Corresponding authors}

*Email: philippe.deniard@cnrs-imn.fr

*Email: christophe.payen@cnrs-imn.fr

\section{Notes}

The authors declare no competing financial interest.

\section{- ACKNOWLEDGMENTS}

The authors are grateful for the beamtime obtained at beamline SAMBA at the SOLEIL synchrotron radiation source, France (project 20141046). We thank Eric Gautron and Nicolas Stephant for their attempts at performing electron microscopy. 


\section{— REFERENCES}

(1) Qu, W.; Wlodarski, W.; Meyer, J.U. Comparative study on micromorphology and humidity sensitive properties of thin-film and thickfilm humidity sensors based on semiconducting $\mathrm{MnWO}_{4}$. Sens. Actuators B 2000, 64, 76-82.

(2) Arkenbout, A. H.; Palstra, T. T. M.; Siegrist, T.; Kimura, T. Ferroelectricity in the Cycloidal Spiral Magnetic Phase of MnWO4. Phys. Rev. B 2oo6, 74, 184431.

(3) Montini, T.; Gombac, V.; Hameed, A.; Felisari, L.; Adami, G.; Fornasiero, P. Synthesis, characterization and photocatalytic performance of transition metal tungstates. Chem. Phys. Lett 2010, 498, 113-119.

(4) Jibril, B. Y. Catalytic Performances and Correlations with Metal Oxide Band Gaps of MetalTungsten Mixed Oxide Catalysts in Propane Oxydehydrogenation. React. Kinet. Catal. Lett. 2005, 86, 171-177.

(5) Zou, Q.; Tang, R.; Zhao, H.; Jiang, J.; Li, J.; Fu, Y. Hyaluronic-Acid-Assisted Facile Synthesis of $\mathrm{MnWO}_{4}$ Single-Nanoparticle for Efficient Trimodal Imaging and Liver-Renal Structure Display ACS Appl. Nano Mater. 2018, 1, 101-110.

(6) Zhang, E.; Xing, Z.; Wang, J.; Ju, Z.; Qian, Y. Enhanced energy storage and rate performance induced by dense nanocavities inside $\mathrm{MnWO}_{4}$ nanobars. RSC Advances 2012, 2, 6748-6751.

(7) Dey, S.; Ricciardo, R.A.; Cuthbert, H.L.; Woodward, P.M. Metal-to-Metal Charge Transfer in $\mathrm{AWO}_{4}(\mathrm{~A}=\mathrm{Mg}, \mathrm{Mn}, \mathrm{Co}, \mathrm{Ni}, \mathrm{Cu}$, or $\mathrm{Zn})$ Compounds with the Wolframite Structure. Inorg. Chem. 2014, 53, 4394-4399.

(8) Sleight, A.W. Accurate Cell Dimensions for $\mathrm{ABO}_{4}$ Molybdates and Tungstates. Acta Cryst.B 1972, 28, 2899-2902.

(9) Kihlborg, L.; Gebert, E. CuWO4, a Distorted Wolframite-Type Structure. Acta Cryst.B 1970, 26, 1020-1026.

(10) Macavei, J.; Schulz, H. The crystal structure of wolframite type tungstates at high pressure. Z.Kristallogr. 1993, 207, 193-208.

(11) Chen, S.-J.; Chen, X.-T.; Xue, Z.; Zhou, J.-H.; Li, J.; Hong, J-M.; You, X.-H. Morphology control of $\mathrm{MnWO}_{4}$ nanocrystals by solvothermal route. J. Mater. Chem. 2003, 13, 1132-1135.

(12) Tong, W.; Li, L..; Hu, W.; Yan, T.; Guan, X.; Li, G. Kinetic control of MnWO4 Nanoparticles for Tailored Structural Properties. J. Phys. Chem. C 2010, 114, 15298-15305.

(13) Yu, S.-H.; Liu, B.; Mo, M.-S.; Huang, J.-H.; Liu, X.-M., and Qian, Y.-T. General Synthesis of Single-Crystal Tungstate Nanorods/Nanowires: A Facile, Low-Temperature Solution Approach. Adv. Funct. Mater. 2003, 13, 639-647.

(14) Ungelenk, J.; Roming, S.; Adler, P.; Schnelle, W.; Winterlik, J.; Felser, C.; Feldmann, C. Ultrafine $\mathrm{MnWO}_{4}$ nanoparticles and their magnetic properties. Solid State Sciences 2015, 46, 89-94.

(15) Patureau, P.; Dessapt, R.; Deniard, P.; Chung, U.-C.; Michau, D.; Josse, M.; Payen, C.; Maglione, M. Persistent Type-II Multiferroicity in Nanostructured $\mathrm{MnWO}_{4}$ Ceramics. Chem. Mater. 2016, 28, 7582-7585.

(16) Kim, B.H.; Hackett, M.J.; Park, J.; Hyeon, T. Synthesis, Characterization, and Application of Ultrasmall Nanoparticles. Chem. Mater. 2o14, 26, 59-71.

(17) Bojesen, E.D.; Iversen, B.B. The chemistry of nucleation. CrystEngComm 2o16, 18, 83328353.

(18) Thongtem, S.; Wannapop, S.; Phuruangrat, A.; Thongtem, T. Cyclic microwave-assisted spray synthesis of nanostructured $\mathrm{MnWO}_{4}$. Materials Letters 2009, 63, 833-836.

(19) Zhou, S.; Huang, J.; Zhang, T.; Ouyang H.; Li, A.; Zhang, Z. Effect of variation Mn/W molar ratios on phase composition, morphology and optical properties of $\mathrm{MnWO}_{4}$. Ceramics International 2013, 39, 5159-5163. 
(20) Petricek, V.; Dusek, M.; Palatinus, L. Crystallographic Computing System JANAzoo6: General Features. Z. Für Krist. 2014, 229, 345-352.

(21) Cheary, R. W.; Coelho, A.A. Axial Divergence in a Conventional X-ray Powder Diffractometer. I. Theoretical Foundations. J. Appl. Crystallogr. 1998, 31, 851-861.

(22) Ravel, B.; Newville, M., ATHENA, ARTEMIS, HEPHAESTUS: data analysis for X-ray absorption spectroscopy using IFEFFIT. Journal of Synchrotron Radiation 2005, 12, 537-541.

(23) Qiu, X.; Thompson, J. W.; Billinge, S. J. PDFgetX2: a GUI-driven program to obtain the pair distribution function from X-ray powder diffraction data. J. Appl. Crystallogr. 2004, 37, 678-678.

(24) Farrow, C. L.; Juhas, P.; Liu, J. W.; Bryndin, D.; Božin, E. S.; Bloch, J.; Proffen, T.; Billinge, S. J. L. PDFfit2 and PDFgui: computer programs for studying nanostructure in crystals. Journal of Physics: Condensed Matter 2007, 19, 335219.

(25) Bain, G. A.; Berry, J.F. Diamagnetic Corrections and Pascal's Constants. J. Chem. Educ. 2008, 85, 532-536.

(26) Krustev, S.; Ivanov, K.; Klissurski, D. Preparation of manganous(II) tungstate by a precipitation method. J. Alloys Compounds 1992, 182, 189-193.

(27) Farrugia, L.J. Sodium tungstate dihydrate: a redetermination. Acta Cryst. 20o7, E63, i142.

(28) Yamazoe, S.; Hitomi, Y.; Shishido T.; Tanaka T. XAFS Study of Tungsten L1- and L3-Edges: Structural Analysis of $\mathrm{WO}_{3}$ Species Loaded on $\mathrm{TiO}_{2}$ as a Catalyst for Photo-oxidation of $\mathrm{NH}_{3}$. J. Phys. Chem. C 2008, 112, 6869-6879.

(29) Farges F. Ab initio and experimental pre-edge investigations of the Mn K-edge XANES in oxide-type materials. Phys. Rev. B 2005, 71, 155109.

(30) Lautenschläger, G.; Weitzel, H.; Vogt, T.; Hock, R.; Böhm, A.; Bonnet, M.; Fuess, H. Magnetic Phase Transitions of $\mathrm{MnWO}_{4}$ Studied by the Use of Neutron Diffraction. Phys. Rev. B 1993, 48, 6087-6098.

(31) Ye, F.; Fishman, R. S.; Fernandez-Baca, J. A.; Podlesnyak, A. A.; Ehlers, G.; Mook, H. A.; Wang, Y.; Lorenz, B.; Chu, C. W. Long-range magnetic interactions in the multiferroic antiferromagnet MnWO4. Phys. Rev. B 2011, 83, 140401. 


\section{Table of Contents Synopsis}

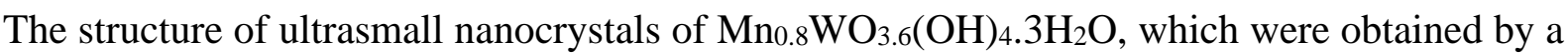
simple salt metathesis reaction involving stoichiometric amounts of manganese(II) chloride and sodium tungstate, was found to be similar to the wolframite structure of bulk-MnWO 4 .

\section{Table of Contents Graphic}

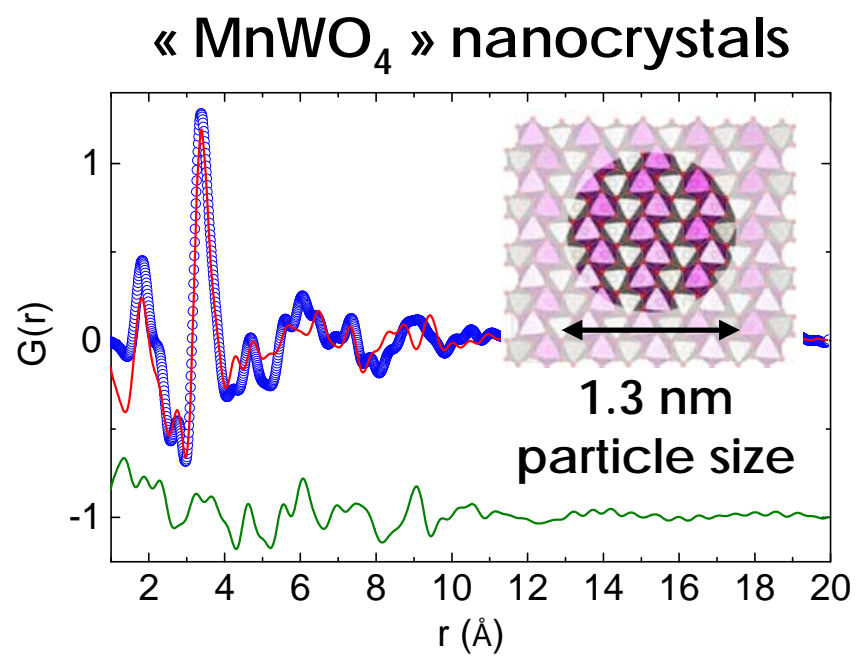

